\section{Response to "The importance of study design in the assessment of nonnutritive sweeteners and cardiometabolic health"}

We thank Sievenpiper and colleagues ${ }^{1}$ for their interest in our work. ${ }^{2}$ Although we agree that study design must be considered when evaluating evidence to inform health decision making, it is often necessary to look beyond randomized controlled trials (RCTs) for additional (and sometimes more appropriate) evidence. Despite their strengths, RCTs can have substantial limitations, including insufficient duration of intervention or follow-up, small sample size or low power, poor external validity owing to enrollment of a highly selected population, reliance on imperfect surrogate outcomes and inability to reliably estimate safety outcomes. ${ }^{3}$ Reflecting these potential weaknesses, the RCTs eligible for our meta-analysis ${ }^{2}$ collectively enrolled only 1003 individuals who were followed for a median of six months. Participants were overweight or had hypertension, and the RCTs focused on short-term changes in body composition rather than cardiovascular disease.

We acknowledged and discussed the limitations of observational studies in our paper, and clearly articulated that despite relatively consistent results across multiple large prospective cohort studies (405907 participants followed for a median of 10 years), most observed associations have not been confirmed in RCTs. However, RCTs published to date have not evaluated the long-term impact of nonnutritive sweeteners (NNS) on cardiometabolic outcomes such as type 2 diabetes, hypertension and cardiovascular disease. This is not surprising, as it would be extremely costly and challenging to enroll a sufficient number of participants, achieve adherence to a long-term dietary intervention and conduct sufficiently long-term follow-up to evaluate these relatively uncommon outcomes that progress slowly over time. Large prospective cohort studies address these challenges and offer a powerful opportunity to pose research questions that cannot be feasibly addressed in RCTs.

We agree that it is important to consider the nature of the comparator in RCTs, and that NNS trials using caloric comparators are useful to evaluate the replacement of sugar with NNS. This is particularly relevant to short-term strategies to reduce sugar intake and lose weight; however, we explicitly aimed to evaluate the long-term effects of routine NNS consumption - a pattern that is increasingly common in the general population, with $40 \%$ of adults and $25 \%$ of children reporting regular NNS consumption. ${ }^{4}$ In this scenario, rather than "replacing sugar," individuals are consuming NNS as a regular component of their habitual diet and thus, "no NNS" (placebo or noncaloric comparator) is a more relevant control.

We disagree with the statement that "the available evidence supports the intended benefits of NNS as being similar to that of other interventions to reduce calories, such as water". ${ }^{1}$ Although evidence from RCTs suggests equivalence to water or other noncaloric comparators for relatively short-term outcomes, evidence from prospective observational studies suggests potentially adverse long-term associations with incident hypertension, stroke and cardiovascular disease. These observational data are not "discordant" with evidence from RCTs because no RCTs to date have reported on these outcomes. In the absence of long-term cardiovascular and safety data, claiming that NNS are analogous to water is unjustified and potentially irresponsible. The absence of short-term adverse effects from RCTs does not establish long-term safety.

We fully agree that further research is needed to clarify the long-term effects of NNS - both synthetic and "natural" evaluated against caloric and noncaloric comparators. Multiple study designs and diverse data sources will be required to address these questions and, in all cases, it will be important to consider study sponsorship and author conflicts of interest as potential sources of bias. ${ }^{5}$
To this end, we are pleased that the Canadian Institutes of Health Research have recognized this important knowledge gap and dedicated public funds to support research on NNS as part of their recent "Sugar and Health" strategic funding opportunity. We look forward to the results of this and other research to establish the long-term risks and benefits of NNS.

\section{Meghan B. Azad PhD}

Assistant Professor, Department of Pediatrics and Child Health, University of Manitoba; Research Scientist, Children's Hospital Research Institute of Manitoba, Winnipeg, Man.

\section{Dylan S. MacKay MSc PhD}

Clinical Trialist, George \& Fay Yee Centre for Healthcare Innovation; Adjunct Professor, Department of Food and Human Nutritional Sciences, University of Manitoba, Winnipeg, Man.

\section{Ryan Zarychanski MD MSc}

Assistant Professor, Department of Internal Medicine; Clinical Scientist, George \& Fay Yee Centre for Healthcare Innovation, University of Manitoba, Winnipeg, Man.

Cite as: CMAJ 2017 November 20;189: E1426. doi: 10.1503/cmaj.733441

\section{References}

1. Sievenpiper JL, Khan TA, Ha V, et al. The importance of study design in the assessment of nonnutritive sweeteners and cardiometabolic health [letter]. CMAJ 2017;189:E1424-5.

2. Azad MB, Abou-Setta AM, Chauhan BF, et al. Nonnutritive sweeteners and cardiometabolic health: a systematic review and meta-analysis of randomized controlled trials and prospective cohort studies. CMAJ 2017;189:E929-39.

3. Frieden TR. Evidence for health decision making - beyond randomized, controlled trials. N Engl J Med 2017;377:465-75.

4. Sylvetsky AC, Jin Y, Clark EJ, et al. Consumption of low-calorie sweeteners among children and adults in the United States. J Acad Nutr Diet 2017; 117:441-8.e2.

5. Mandrioli D, Kearns CE, Bero LA. Relationship between research outcomes and risk of bias, study sponsorship, and author financial conflicts of interest in reviews of the effects of artificially sweetened beverages on weight outcomes: a systematic review of reviews. PLoS One 2016; 11:e0162198.

Competing interests: None declared. 